\title{
THE LARGEST LINEAR HOMOGENEOUS GROUP WITH AN INVARIANT PFAFFIAN.
}

\author{
BY DR. L. E. DICKSON.
}

(Read before the American Mathematical Society at the Meeting of October $29,1898$. )

1. In the December number of the Bulletin (pp. 120135) I have shown that the second compound of the general $2 m$-ary linear homogeneous group is a linear group in $C_{z m, 2} \equiv m(2 m-1)$ variables which leaves invariant the Pfaffian

$$
F \equiv[1,2, \cdots, 2 m] .
$$

Denoting the variables as follows:

$$
Y_{i j} \equiv-Y_{j i} \quad(i, j=1, \cdots, 2 m ; i \neq j),
$$

the second compound was proved to contain exactly $(2 \mathrm{~m})^{2}$ linearly independent infinitesimal transformations

$$
\sum_{r \neq s, t}^{r=1, \cdots, 2 m} Y_{r t} \frac{\partial f}{\partial Y_{r s}} \delta t . \quad(t, s=1, \cdots, 2 m) .
$$

The object of the present note is to prove that the largest linear homogeneous group $G$ in the $m(2 m-1)$ variables $(1)$ which leaves invariant the Pfaffian $F$ contains only the $(2 m)^{2}$ linearly independent transformations (2).

2. Let the general infinitesimal transformation of the group $G$ be as follows:

$$
\delta Y_{i j}=\sum_{k \neq l}^{k=1, \cdots, 2 m} \alpha_{k l}^{i j} Y_{k l} \delta t \quad(i, j=1, \cdots, 2 m ; i \neq j),
$$

where, on account of (1), we may suppose

$$
\alpha_{k l}^{i j}=-\alpha_{l l k}^{i j}=+\alpha_{l k}^{j i} .
$$

The condition that (3) shall multiply $F$ by a constant $c \delta t$ is as follows :

$$
\sum_{i, j, k, l} \frac{\partial F}{\partial Y_{i l}^{-}} \alpha_{k l}^{i j} Y_{k l}=c F .
$$

Now 


$$
\begin{aligned}
\frac{\partial F}{\partial Y_{i j}} & =\frac{\partial}{\partial Y_{i j}}\left\{(-1)^{i+j-1}[i j 12 \cdots i-1 i+1 \cdots j-1 j+1 \cdots 2 m]\right. \\
& =(-1)^{i+\jmath-1}[12 \cdots i-1 i+1 \cdots j-1 j+1 \cdots 2 m] .
\end{aligned}
$$

Comparing the coefficients of the terms in (5) of the type

$$
(-1)^{\sigma} Y_{i_{1} i_{2}} Y_{i_{3} i_{4}} \cdots Y_{i_{2 m-1} i_{2 m}},
$$

where $i_{1}, i_{2}, \cdots, i_{2 m}$ is a permutation of $1,2, \cdots, 2 m$ and where $\sigma$ denotes the number of transpositions giving that permutation, we obtain the conditions

$$
\alpha_{i_{1} i_{2}}^{i_{1} i_{2}}+\alpha_{i_{3} i_{4}}^{i_{3} i_{4}}+\cdots+\alpha_{i_{2 m}=1 i_{2 m}}^{i_{2} m}=c .
$$

Comparing the coefficients of the terms,

$$
Y_{i_{3} i_{4}}^{2} Y_{i_{5} i_{6}} \cdots Y_{i_{2 m-1} i_{2 m}}
$$

we obtain the conditions

$$
\alpha_{i_{3} i_{4}}^{i_{1} i_{2}}=0 \quad\left(i_{3} \text { and } i_{4} \neq i_{1} \text { or } i_{2}\right) .
$$

Comparing the coefficients of the terms

we find

$$
Y_{i_{1} i_{3}} Y_{i_{3} i_{4}} \cdots Y_{i_{2 m-1} i_{2 m}},
$$

$$
\alpha_{i_{1} i_{3}}^{i_{1} i_{2}}-\alpha_{i_{4} i_{3}}^{i_{1} i_{2}}=0
$$

$\left(i_{1}, i_{2}, i_{3}, i_{4}\right.$, being any four different integers $\left.\equiv 2 m\right)$.

We may now obtain a complete set of linearly independent infinitesimal transformations (3), which leave $F$ invariant. According as every $\alpha_{i_{3} i_{4}}^{i_{1} i_{2}}$ is zero, or not every such $\alpha$ is zero, we obtain two independent types of transformations (3), which together form the desired complete set. We consider the two types in succession :

(a) If any $\alpha_{r t}^{r s} \neq 0$, say $=1$, where $r, s, t$ are distinct integers $\equiv 2 m$, then by (8) we have

$$
a_{r t}^{r s}=1 \quad(r=1, \cdots, 2 m ; r \neq s, t) .
$$

Setting every other $\alpha=0$, we obtain a set of solutions of (6), (7), (8), for which

$$
\begin{cases}\delta Y_{r s}=Y_{r t} \delta t & (r=1, \cdots, 2 m ; r \neq s, t) \\ \delta Y_{i j}=0 & (i, j=1, \cdots, 2 m ; i \neq r) .\end{cases}
$$


We thus obtain the $2 m(2 m-1)$ infinitesimal transformations (included in the formula (2))

$$
\sum_{r \neq s, t}^{r=1, \cdots, 2 m} Y_{r t} \frac{\partial f}{\partial Y_{r s}} \delta t \quad\left(\begin{array}{c}
s, t=1, \cdots, 2 m \\
s \neq t
\end{array}\right),
$$

which are therefore linearly independent.

(b) If next

$$
a_{r t}^{r s}=0 \quad(r, s, t=1, \cdots, 2 m ; r+s,+t),
$$

the general transformation (3) becomes

$$
\delta Y_{i j}=a_{i j}^{i i} Y_{i j} \delta t \quad(i, j=1, \cdots, 2 m),
$$

where the $a_{i j}^{i j}$ are subject to the conditions (6).

Writing for brevity [see (4)],

$$
a_{i j}^{i j} \equiv(i j)=(j i),
$$

these conditions (6) become

$$
\left(i_{1} i_{2}\right)+\left(i_{3} i_{4}\right)+\cdots+\left(i_{2 m-1} i_{2 m}\right)=c .
$$

We obtain at once the following $2 m$ sets of solutions of these equations, each set being given by one value of $l$ chosen from $1,2, \cdots, 2 m$;

(10) $\begin{aligned}(l 1)=(l 2)=\cdots=(l l-1)= & (l l+1)=\cdots=(l 2 m)=c \\ (i j)=0 & {[i, j=1, \cdots, 2 m ; i \neq l] . }\end{aligned}$

These sets of solutions of the equations (6), (7), (8) give rise to the following $2 m$ infinitesimal transformations :

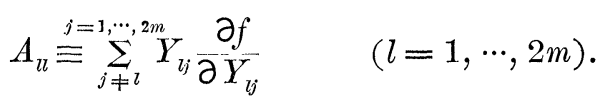

These transformations are linearly independent if $m \equiv 2$. Indeed, if

$$
\sum_{l=1}^{2 m} k_{t} A_{l l}=0,
$$

upon equating the coefficients of $\frac{\partial f}{\partial Y_{r s}}$ in the two members, we have

$$
k_{r}+k_{s}=0 \quad(r, s=1, \cdots, 2 m ; r \neq s) .
$$

Hence, if $m \equiv 2, k_{l}=0(l=1, \cdots, 2 m)$. 
The transformations (9) and (11) make up the $(2 m)^{2}$ linearly independent transformations (2). It follows from the theorem of the next paragraph that there do not exist more than $2 m$ linearly independent transformations of the type $(b)$. We will then have proved the following theorem :

The largest linear homogeneous group in $C_{2 m, 2}$ variables leaving invariant the Pfaffian $[1,2, \cdots, 2 m]$ is identical with the second compound of the general m-ary linear homogeneous group.

3. Theorem. The $m(2 m-1)$ quantities

$$
(i j) \equiv(j i) \quad[i, j=1,2, \cdots, 2 m, i \neq j]
$$

satisfying the $1 \cdot 3 \cdot 5 \cdots(2 m-3)(2 m-1)$ equations

$$
\left[E_{2 m}\right] \quad\left(i_{1} i_{2}\right)+\left(i_{3} i_{4}\right)+\cdots+\left(i_{2 m-1} i_{2 m}\right)=c_{2 m}
$$

can all be expressed in terms of certain $2 m$ of the ( $i j)$, for example, $\left[Q_{2 m}\right]\left\{\left(\begin{array}{ll}12 \\ 12\end{array}\right)(34),(56), \cdots,(2 l-12 l), \cdots,(2 m-12 m)\right.$;

but not in terms of fewer than $2 m$ of them if $m>1$.

The last part of the theorem follows from the linear independence of the $2 m$ infinitesimal transformation of type (b) above.

The first part of the theorem will be proved by induction. For $m=2$, it is evident; for the equations $\left[E_{4}\right]$ are as follows:

$$
(12)+(34)=(13)+(24)=(14)+(23)=c_{4} \text {. }
$$

Supposing the first part of the theorem to be true for a given value of $2 m$, we can prove it true for the next value $2(m+1)$. Indeed, applying this hypothesis to certain equations of the set $\left[E_{2 m+2}\right]$, viz. :

$$
\left(i_{1} i_{2}\right)+\left(i_{3} i_{4}\right)+\cdots+\left(i_{2 m-1} i_{2 m}\right)=c_{2 m+2}-(2 m+12 m+2),
$$

where $i_{1}, i_{2}, \cdots, i_{2 m}$ is a permutation of $1,2, \cdots, 2 m$, it follows that the quantities

$$
\text { (ij) } \quad[i, j=1,2, \cdots, 2 m ; i \neq j]
$$

can be expressed in terms of the quantities $Q_{2 m}$ and that $c_{2 m+2}$ is expressible in terms of the quantities $Q_{2 m}$ together with $(2 m+12 m+2)$.

Consider next the equations of the set $\left[E_{2 m+2}\right]$

$$
\begin{gathered}
\left(i_{1} i_{2}\right)+\left(i_{3} i_{4}\right)+\cdots+\left(i_{2 m-3} i_{2 m-2}\right)+(j 2 m+1) \\
+(2 m 2 m+2)=c_{2 m+2}
\end{gathered}
$$


where $i_{1}, i_{2}, \cdots, i_{2 m-2}, j$ form a permutation of $1,2, \cdots, 2 m-1$. It follows that every

$$
(j 2 m+1) \quad[j=1,2, \cdots, 2 m-1]
$$

is expressible in terms of the $Q_{2 m}, c_{2 m+2}$ and $(2 m 2 m+2)$ and hence in terms of the $Q_{2 m+2}$.

From the equation

$$
\begin{aligned}
& (12)+(34)+\cdots+(2 m-52 m-4)+(2 m-32 m) \\
& +(2 m-22 m+1)+(2 m-12 m+2)=c_{2 m+2}
\end{aligned}
$$

we have $(2 m-12 m+2)$ expressed in terms of the quantities $Q_{2 m+2}$ (by using our earlier results). Hence, from the equation

$$
\begin{gathered}
(12)+(34)+\cdots+(2 m-32 m-2)+(2 m 2 m+1) \\
+(2 m-12 m+2)=c_{2 m+2},
\end{gathered}
$$

we obtain $(2 m 2 m+1)$ expressed in terms of the $Q_{2 m+2}$. We have, therefore, every

$$
(j 2 m+1) \quad[j=1,2, \cdots, 2 m+2]
$$

expressed in terms of the $Q_{2 m+2}$.

Finally, from the equations

$$
\begin{gathered}
\left(i_{1} i_{2}\right)+\cdots+\left(i_{2 m-1} i_{2 m}\right)+(j 2 m+2) \\
+(2 m-12 m+1)=c_{2 ! m+2}
\end{gathered}
$$

where $j, i_{1}, i_{2}, \cdots, i_{2 m}$ form a permutation of $1,2, \cdots, 2 m-2$, $2 m$, we are able to express

$$
(j 2 m+2) \quad[j=1,2, \cdots, 2 m-2,2 m]
$$

in terms of the $Q_{2 m+2}$.

Combining our results, we find that every

$$
\text { ( } i j) \quad[i, j=1,2, \cdots, 2 m+2 ; i \neq j]
$$

is expressible in terms of the quantities $Q_{2 m+2}$.

University of California, September 23, 1898. 